\title{
A SPECTRAL STUDY OF AN INFINITE AXISYMMETRIC ELASTIC LAYER
}

\author{
LAHCÈNE CHORFI $^{1}$
}

\begin{abstract}
We present here a theoretical study of eigenmodes in axisymmetric elastic layers. The mathematical modelling allows us to bring this problem to a spectral study of a sequence of unbounded self-adjoint operators $A_{n}, n \in \mathbb{N}$, in a suitable Hilbert space. We show that the essential spectrum of $A_{n}$ is an interval of type $[\gamma,+\infty[$ and that, under certain conditions on the coefficients of the medium, the discrete spectrum is non empty.
\end{abstract}

Résumé. Nous présentons ici une étude théorique des modes propres dans une couche élastique axisymétrique. La modélisation mathématique permet de ramener ce problème à l'étude spectrale d'une suite d'opérateurs $A_{n}, n \in \mathbb{N}$, non bornés et autoadjoints dans un espace de Hilbert adéquat. On montre que le spectre essentiel de $A_{n}$ est un intervalle du type $[\gamma,+\infty[$ et que, sous certaines conditions portant sur les coefficients du milieu, le spectre discret est non vide.

Mathematics Subject Classification. 35P15, 47A70, 73D30.

Received: October 23, 2000. Revised: May 5, 2001.

\section{The PRoblem Setting}

We consider an elastic layer occupying the open set $\tilde{\Omega}=\left\{\left(x_{1}, x_{2}, x_{3}\right) \in \mathbb{R}^{3} ; 0<x_{3}<h\right\}$. We assume that the medium is axisymmetric, which means that the density $\rho$ and the Lamé coefficients $(\lambda, \mu)$ depend on $(r, z)$ if $r<\mathrm{R}$, and are constants if $r>\mathrm{R}$ (the triple $(r, \theta, z)$ denote the cylindrical coordinates). We suppose that the surface $z=0$ is rigid and the one at $z=h$ is stress free. We call an eigenmode a field of displacement $U(x, t)$, time-harmonic, travelling in the medium without source for $t>0$, and with the amplitude $|U| \in L^{2}(\tilde{\Omega})$ (i.e. the energy of the mode is localized in a neighbourhood of the axis of symmetry).

In this paper we consider the question of existence of such eigenmodes which amounts to studying the spectrum of a sequence of self-adjoint operators $A_{n}$ derived from the linearized elasticity equations $(n$ is the order of the harmonic). Generalized eigenfunction expansion of $A_{n}$ is essential for the construction of the Green's function which enable us to resolve transient problems (with source). This problem appears, for example, in geophysics in the study of seismic waves generated in soil by a source placed in a borehole (with variable diameter and/or with casing) (cf. $[3,13])$.

This paper is a generalisation of [4] where we studied the guided waves in an elastic space with a symmetry of revolution, in other words the coefficients depend only on the radial variable $r$. The spectral theory of self-adjoint operators and in particular the Min-Max principle are the main tools used (as in $[1,2,5])$.

Keywords and phrases. Elasticity, axisymmetry, eigenmodes, min-max principle.

1 Département de Mathématiques, Université Badji Mokhtar de Annaba, BP 12, 23000 Annaba, Algeria. 
Our problem appears to be more difficult than those mentioned above. Indeed, in our case the free boundary does not permit us to extend directly the results of [1], moreover as the medium is heterogeneous with unbounded boundary, we cannot also extend the result of [2] and [5].

The main result of this paper is the determination of the essential spectrum of the operator $A_{n}$. Then we establish two existence results of eigenmodes for suitable Lamé coefficients.

Our paper is organized as follows. In Section 2, we obtain the equations for eigenmodes. Section 3 is devoted to the mathematical study of the problem. As we shall see, an eigenmode appears as an eigenfunction of an unbounded self-adjoint operator $A_{n}$ (with two variables) defined in an adequate Hilbert space. In Section 4 , we establish the regularity of $u$, the solution of $A_{n} u=f$ in the exterior domain $\left.\Omega_{\mathrm{R}}^{\prime}=\right] \mathrm{R},+\infty[\times] 0, h[$. In Section 5 , we study $\sigma_{e}\left(A_{n}\right)$ which is the essential spectrum of the operators $A_{n}$. This is a fundamental preliminary step in the analysis of their point spectrum. The value of the lower bound of $\sigma_{e}\left(A_{n}\right)$ seems predictable but its proof is not so much trivial. Indeed, as the free surface is unbounded, this leads to significant difficulties in elasticity. To overcome these difficulties, we introduce weighted Sobolev spaces, which is not classic in the previous works on waveguides, to obtain a compactness result. In Section 6, we give sufficient existence conditions of eigenmodes for the operator $A_{n}$. Here we point out the influence of the perturbation.

\section{The EQUATIONS}

Because the structure of the layer is cylindrical, we are looking for a particular solution $U(x, t)$ of the form:

$$
\left\{\begin{array}{l}
U(r, \theta, z, t)=U_{r} e_{r}+U_{\theta} e_{\theta}+U_{z} e_{z} \quad \text { with } \\
\left(U_{r}, U_{\theta}, U_{z}\right)=\left(u_{1}(r, z),-i u_{2}(r, z), u_{3}(r, z)\right) \exp i(n \theta \pm \omega t), \quad n \in \mathbb{Z},
\end{array}\right.
$$

where $\left(e_{r}, e_{\theta}, e_{z}\right)$ is the local basis of the cylindrical coordinates $(r, \theta, z)$. The field $U(r, \theta, z, t)$ must satisfy the elastodynamic equations (in cylindrical coordinates) [6]:

$$
\left\{\begin{aligned}
\frac{\partial\left(r \sigma_{r r}\right)}{\partial r}+\frac{\partial \sigma_{\theta r}}{\partial \theta}+r \frac{\partial \sigma_{z r}}{\partial z}-\sigma_{\theta \theta} & =r \rho \frac{\partial^{2} U_{r}}{\partial t^{2}} \\
\frac{1}{r} \frac{\partial\left(r^{2} \sigma_{r \theta}\right)}{\partial r}+\frac{\partial \sigma_{\theta \theta}}{\partial \theta}+r \frac{\partial \sigma_{\theta z}}{\partial z} & =r \rho \frac{\partial^{2} U_{\theta}}{\partial t^{2}} \\
\frac{\partial\left(r \sigma_{r z}\right)}{\partial r}+\frac{\partial \sigma_{\theta z}}{\partial \theta}+r \frac{\partial \sigma_{z z}}{\partial z} & =r \rho \frac{\partial^{2} U_{z}}{\partial t^{2}}
\end{aligned}\right.
$$

where $\sigma$ is the stress tensor given by Hooke's law:

$$
\left\{\begin{array}{lll}
\sigma_{r r}=\lambda \operatorname{div} U+2 \mu \frac{\partial U_{r}}{\partial r} \quad ; & \sigma_{r \theta}=\mu\left[\frac{1}{r} \frac{\partial U_{r}}{\partial \theta}+r \frac{\partial}{\partial r}\left(\frac{U_{\theta}}{r}\right)\right] \\
\sigma_{r z}=\mu\left(\frac{\partial U_{z}}{\partial r}+\frac{\partial U_{r}}{\partial z}\right) ; & \sigma_{\theta \theta}=\lambda \operatorname{div} U+\frac{2 \mu}{r}\left(\frac{\partial U_{\theta}}{\partial \theta}+U_{r}\right) \\
\sigma_{\theta z}=\mu\left(\frac{\partial U_{\theta}}{\partial z}+\frac{1}{r} \frac{\partial U_{z}}{\partial \theta}\right) ; & \sigma_{z z}=\lambda \operatorname{div} U+2 \mu \frac{\partial U_{z}}{\partial z}
\end{array}\right.
$$

with

and the boundary conditions

$$
\operatorname{div} U=\frac{1}{r}\left[\frac{\partial\left(r U_{r}\right)}{\partial r}+\frac{\partial U_{\theta}}{\partial \theta}\right]+\frac{\partial U_{z}}{\partial z}
$$

$$
U=0, \text { if } z=0 \text { and } \sigma_{r z}(U)=\sigma_{r \theta}(U)=\sigma_{z z}(U)=0 \text {, if } z=h .
$$


We denote by $u(r, z)=\left(u_{1}, u_{2}, u_{3}\right)$ the field of amplitudes. Therefore, to find the eigenmodes we must solve, for each $n \in \mathbb{Z}$, the following two dimensional problem:

$$
\left\{\begin{array}{l}
\text { Find } u \in \mathcal{D}^{\prime}(\Omega), u \neq 0, \text { and } \omega>0 \text { such that : } \\
\sqrt{r}|u| \in L^{2}(\Omega) \text { with } \Omega=\left\{(r, z) \in \mathbb{R}^{2}, r>0,0<z<h\right\} ; \\
\mathcal{A}_{n} u=\omega^{2} u \quad \text { in } \Omega ; \\
u(r, 0)=0 ; \quad \sigma_{r z}^{n}(u)(r, h)=\sigma_{\theta z}^{n}(u)(r, h)=\sigma_{z z}^{n}(u)(r, h)=0 .
\end{array}\right.
$$

where $\mathcal{A}_{n}$ is the partial differential operator defined formally by:

$$
\begin{aligned}
\mathcal{A}_{n} u= & \frac{1}{\rho r}\left[-\frac{\partial}{\partial r}\left(B \frac{\partial u}{\partial r}+B_{2} u\right)+B_{1} \frac{\partial u}{\partial r}+B_{3} u-\frac{\partial}{\partial r}\left(B_{6} \frac{\partial u}{\partial z}\right)\right. \\
& \left.-\frac{\partial}{\partial z}\left(B_{7} \frac{\partial u}{\partial r}\right)-\frac{\partial}{\partial z}\left(B_{8} \frac{\partial u}{\partial z}\right)+B_{4} \frac{\partial u}{\partial z}-\frac{\partial}{\partial z}\left(B_{5} u\right)\right]
\end{aligned}
$$

and the matrices $B$ and $B_{i}, i=1$ to 8 , are given by:

$$
\begin{gathered}
B=\left(\begin{array}{ccc}
(\lambda+2 \mu) r & 0 & 0 \\
0 & \mu r & 0 \\
0 & 0 & \mu r
\end{array}\right) \quad ; \quad B_{1}=B_{2}^{t}=\left(\begin{array}{ccc}
\lambda & -n \mu & 0 \\
n \lambda & -\mu & 0 \\
0 & 0 & 0
\end{array}\right) \\
\left.B_{3}=\left(\begin{array}{ccc}
\frac{\lambda+\left(n^{2}+2\right) \mu}{r} & \frac{n(\lambda+3 \mu)}{r} & 0 \\
\frac{n(\lambda+3 \mu)}{r} & \frac{n^{2} \lambda+\left(2 n^{2}+1\right) \mu}{r} & 0 \\
0 & 0
\end{array}\right) \quad \begin{array}{c}
n^{2} \mu \\
r
\end{array}\right) \quad B_{4}=B_{5}^{t}=\left(\begin{array}{ccc}
0 & 0 & \lambda \\
0 & 0 & n \lambda \\
0 & -n \mu & 0
\end{array}\right) \\
B_{6}=B_{7}^{t}=\left(\begin{array}{ccc}
0 & 0 & \lambda r \\
0 & 0 & 0 \\
\mu r & 0 & 0
\end{array}\right) \quad ; \quad B_{8}=\left(\begin{array}{ccc}
\mu r & 0 & 0 \\
0 & \mu r & 0 \\
0 & 0 & (\lambda+2 \mu) r
\end{array}\right) .
\end{gathered}
$$

The reduced stress vector $\sigma_{z}^{n}(u)$ is given by:

$$
\left\{\begin{array}{l}
\sigma_{r z}^{n}(u)=\mu\left(\frac{\partial u_{3}}{\partial r}+\frac{\partial u_{1}}{\partial z}\right) \\
\sigma_{\theta z}^{n}(u)=\mu\left(\frac{\partial u_{2}}{\partial z}-\frac{n}{r} u_{3}\right) \\
\sigma_{z z}^{n}(u)=(\lambda+2 \mu) \frac{\partial u_{3}}{\partial z}+\lambda\left(\frac{\partial u_{1}}{\partial r}+\frac{u_{1}+n u_{2}}{r}\right)
\end{array}\right.
$$

\section{Remark 2.1.}

1) We have introduced $(-i)$ in the solution (1) to deal with a system with real coefficients.

2) We point out that if the field $u=\left(u_{1}, u_{2}, u_{3}\right)$ satisfies $\left(\tilde{P}_{n}\right)$ then the field $u=\left(u_{1},-u_{2}, u_{3}\right)$ satisfies $\left(\tilde{P}_{-n}\right)$. Therefore it is sufficient to study the problem $\left(\tilde{P}_{n}\right)$ for $n \in \mathbb{N}$.

3) The system of solutions of the form (1) is complete since each solution of (2) can be expanded in series of solutions of type (1). This can be rigorously proved by using Fourier decomposition in $\theta$ (see [10] for $\tilde{\Omega}$ a bounded axisymmetric domain). 


\section{The VARiational Formulation of $\tilde{P}_{n}$}

In the following we suppose that $\lambda, \mu, \rho \in L^{\infty}(\Omega)$ and satisfy the assumptions:

(i) $\exists \mathrm{R}>0$ such that $(\lambda(r, z), \mu(r, z), \rho(r, z))=\left(\lambda_{\infty}, \mu_{\infty}, \rho_{\infty}\right)$ for all $r>\mathrm{R}$,

(ii) $\inf _{\Omega} \lambda(r, z)=\lambda_{-}>0, \inf _{\Omega} \mu(r, z)=\mu_{-}>0, \inf _{\Omega} \rho(r, z)=\rho_{-}>0$.

We also define the velocities of the $P$ and $S$ waves given respectively by

$$
c_{S}=\left(\frac{\mu_{\infty}}{\rho_{\infty}}\right)^{\frac{1}{2}} \text { and } c_{P}=\left(\frac{\lambda_{\infty}+2 \mu_{\infty}}{\rho_{\infty}}\right)^{\frac{1}{2}} \text {. }
$$

We set $\lambda_{+}=\sup _{\Omega} \lambda(r, z), \mu_{+}=\sup _{\Omega} \mu(r, z)$ and $\rho_{+}=\sup _{\Omega} \rho(r, z)$.

We define the real Hilbert space:

$$
H=\left\{u=\left(u_{1}, u_{2}, u_{3}\right) \in\left(L_{l o c}^{2}(\Omega)\right)^{3} ; \sqrt{r}|u| \in L^{2}(\Omega)\right\}
$$

with the inner product $(u, v)=\iint_{\Omega} u v \rho r \mathrm{~d} r \mathrm{~d} z$.

We then define a sequence of real Hilbert spaces:

$$
V_{n}=\left\{u \in\left(H_{l o c}^{1}(\Omega)\right)^{3} \text { such that }\|u\|_{V_{n}}<+\infty \text { and } u(r, 0)=0\right\}, \quad n \in \mathbb{N},
$$

with the norms:

$$
\|u\|_{V_{n}}^{2}=\left\{\begin{array}{l}
\iint_{\Omega}\left[r\left(|u|^{2}+|\nabla u|^{2}\right)+\frac{n^{2}+1}{r}\left(u_{1}^{2}+u_{2}^{2}\right)+\frac{n^{2} u_{3}^{2}}{r}\right] \mathrm{d} r \mathrm{~d} z \quad \text { if } n \neq 1, \\
\iint_{\Omega}\left[r\left(|u|^{2}+|\nabla u|^{2}\right)+\frac{1}{r}\left(u_{1}+u_{2}\right)^{2}+\frac{u_{3}^{2}}{r}\right] \mathrm{d} r \mathrm{~d} z \quad \text { if } n=1 .
\end{array}\right.
$$

Consider the following bilinear form on $V_{n} \times V_{n}$ defined by:

$$
\begin{aligned}
a_{n}(u, v)= & \iint_{\Omega}\left[B \frac{\partial u}{\partial r} \cdot \frac{\partial v}{\partial r}+B_{1} \frac{\partial u}{\partial r} \cdot v+u \cdot B_{1} \frac{\partial v}{\partial r}+B_{3} u \cdot v+\frac{\partial u}{\partial r} \cdot B_{6} \frac{\partial v}{\partial z}\right. \\
& \left.+B_{6} \frac{\partial u}{\partial z} \cdot \frac{\partial v}{\partial r}+u \cdot B_{4} \frac{\partial v}{\partial z}+B_{4} \frac{\partial u}{\partial z} \cdot v+B_{8} \frac{\partial u}{\partial z} \cdot \frac{\partial v}{\partial z}\right] \mathrm{d} r \mathrm{~d} z .
\end{aligned}
$$

The variational formulation of $\left(\tilde{P}_{n}\right)$ is:

$$
\text { Find } u \in V_{n}, u \neq 0 \text {, and } \omega>0 \text { such that: } a_{n}(u, v)=\omega^{2}(u, v), \quad \forall v \in V_{n} \text {. }
$$

The bilinear form $a_{n}(.,$.$) is obviously symmetric and continuous, we shall show that it is coercive and, con-$ sequently, it defines a self-adjoint operator $A_{n}$ in $H$. Then the problem $\left(\mathcal{P}_{n}\right)$ is equivalent to the spectral problem:

$$
\text { Find } u \in D\left(A_{n}\right), u \neq 0 \text {, and } \omega>0 \text { such that: } A_{n} u=\omega^{2} u \text {. }
$$

\section{The coerciveness of $a_{n}(.,$.}

We have the following decomposition:

$$
a_{n}(u, u)=\iint_{\Omega} \lambda\left|\operatorname{div}^{n} u\right|^{2} r \mathrm{~d} r \mathrm{~d} z+\iint_{\Omega} \mu \varepsilon_{n}(u, u) r \mathrm{~d} r \mathrm{~d} z
$$


where

$$
\operatorname{div}^{n} u=\frac{\partial u_{1}}{\partial r}+\frac{\partial u_{3}}{\partial z}+\frac{u_{1}+n u_{2}}{r} \quad, \quad \varepsilon_{n}(u, u)=2 \sum_{\tau, \sigma} \varepsilon_{\tau \sigma}^{n}(u)^{2}
$$

with $\varepsilon^{n}(u)$ the symmetric tensor

$$
\begin{array}{ccrl}
\varepsilon_{r r}^{n}(u)=\frac{\partial u_{1}}{\partial r} ; & \varepsilon_{z z}^{n}(u)=\frac{\partial u_{3}}{\partial z} ; & \varepsilon_{\theta \theta}^{n}(u)=\frac{n u_{2}+u_{1}}{r} ; \\
2 \varepsilon_{r z}^{n}(u)=\frac{\partial u_{3}}{\partial r}+\frac{\partial u_{1}}{\partial z} ; & 2 \varepsilon_{r \theta}^{n}(u)=\frac{n u_{1}+u_{2}}{r}-\frac{\partial u_{2}}{\partial r} ; & 2 \varepsilon_{\theta z}^{n}(u)=\frac{\partial u_{2}}{\partial z}-\frac{n u_{3}}{r} .
\end{array}
$$

To prove the coerciveness of $a_{n}$ it suffices to establish the following inequality

$$
\iint_{\Omega} \varepsilon_{n}(v, v) r \mathrm{~d} r \mathrm{~d} z \geq K\|v\|_{V_{n}(\Omega)}^{2}, \quad \forall v \in V_{n} \text { with } K>0 .
$$

For this we use Korn's inequality in the half-space $\Pi_{h}:=\left\{x \in \mathbb{R}^{3}, x_{3}<h\right\}$ (cf. [9]):

$$
\iiint_{\Pi_{h}} \sum_{i, j=1}^{3}\left|e_{i j}(\tilde{u})\right|^{2} \mathrm{~d} x \geq C_{1} \iiint_{\Pi_{h}}|\nabla \tilde{u}|^{2} \mathrm{~d} x, \quad \text { for } \tilde{u} \in H^{1}\left(\Pi_{h}\right)^{3} \text { with } C_{1}>0 .
$$

The inequality (14) is also valid for $\tilde{u} \in W_{0}^{1}(\tilde{\Omega}):=\left\{\tilde{v} \in H^{1}(\tilde{\Omega})^{3} ;\left.\tilde{v}\right|_{z=0}=0\right\}$.

We would like now to derive (13) from (14). We proceed in two steps.

Step 1. We recall some transformations between Cartesian and cylindrical coordinates (cf. [10]). Each vector field $\tilde{u}=\left(\tilde{u}_{1}, \tilde{u}_{2}, \tilde{u}_{3}\right)$ is transformed into a vector field $u=\left(u_{r}, u_{\theta}, u_{z}\right)$ on $\tilde{\Omega}$ by

$$
u_{r}=\tilde{u}_{1} \cos \theta+\tilde{u}_{2} \sin \theta, \quad u_{\theta}=-\tilde{u}_{1} \sin \theta+\tilde{u}_{2} \cos \theta, \quad u_{z}=\tilde{u}_{3} .
$$

We get the following integrals

$$
\begin{aligned}
\iiint_{\tilde{\Omega}}|\nabla \tilde{u}|^{2} \mathrm{~d} x= & \iiint_{\tilde{\Omega}}\left\{\sum_{\tau=r, \theta, z} r\left(\left|\frac{\partial u_{\tau}}{\partial r}\right|^{2}+\left|\frac{\partial u_{\tau}}{\partial z}\right|^{2}\right)\right. \\
& \left.+\frac{1}{r}\left[\left(\frac{\partial u_{r}}{\partial \theta}-u_{\theta}\right)^{2}+\left(\frac{\partial u_{\theta}}{\partial \theta}+u_{r}\right)^{2}+\left(\frac{\partial u_{z}}{\partial \theta}\right)^{2}\right]\right\} \mathrm{d} r \mathrm{~d} \theta \mathrm{d} z \\
& \iiint_{\tilde{\Omega}} \sum_{i, j=1}^{3}\left|e_{i j}(\tilde{u})\right|^{2} \mathrm{~d} x=2 \iiint_{\tilde{\Omega}} \sum_{\tau, \sigma}\left|\varepsilon_{\tau, \sigma}(u)\right|^{2} r \mathrm{~d} r \mathrm{~d} \theta \mathrm{d} z
\end{aligned}
$$

where

$$
\begin{gathered}
\varepsilon_{r r}(u)=\frac{\partial u_{r}}{\partial r}, \quad \varepsilon_{\theta \theta}(u)=\frac{1}{r}\left(u_{r}+\frac{\partial u_{\theta}}{\partial \theta}\right), \quad \varepsilon_{z z}(u)=\frac{\partial u_{z}}{\partial z}, \\
2 \varepsilon_{r z}(u)=\frac{\partial u_{r}}{\partial z}+\frac{\partial u_{z}}{\partial r}, 2 \varepsilon_{\theta r}(u)=\frac{1}{r} \frac{\partial u_{r}}{\partial \theta}+\frac{\partial u_{\theta}}{\partial r}-\frac{u_{\theta}}{r}, 2 \varepsilon_{z \theta}(u)=\frac{\partial u_{\theta}}{\partial z}+\frac{1}{r} \frac{\partial u_{z}}{\partial \theta} .
\end{gathered}
$$

Step 2. We consider test functions $u^{(n)}, n \in \mathbb{N}$, such that:

$$
u^{(n)}(r, \theta, z)=\left(v_{1}(r, z) \cos n \theta, v_{2}(r, z) \sin n \theta, v_{3}(r, z) \cos n \theta\right)
$$


where $v=\left(v_{1}, v_{2}, v_{3}\right)$ is an arbitrary field in $V_{n}$. The vector field $\tilde{u}^{(n)}$ associated to $u^{(n)}$ by the inverse transformation (15) belongs to the space $W_{0}^{1}(\tilde{\Omega})$.

Inserting the test functions $\tilde{u}^{(n)}$ in (14), and taking into consideration (16) and (17), we derive the estimate

$$
\begin{aligned}
\iint_{\Omega} \varepsilon_{n}(v, v) r \mathrm{~d} r \mathrm{~d} z \geq & C_{1} \iint_{\Omega}\left\{\sum_{i=1}^{3} r\left(\left|\frac{\partial v_{i}}{\partial r}\right|^{2}+\left|\frac{\partial v_{i}}{\partial z}\right|^{2}\right)\right. \\
& \left.+\frac{1}{r}\left[\left(n^{2}+1\right)\left(v_{1}^{2}+v_{2}^{2}\right)+n^{2} v_{3}^{2}+4 n v_{1} v_{2}\right]\right\} \mathrm{d} r \mathrm{~d} z .
\end{aligned}
$$

To conclude, we use the inequality

$$
\left(n^{2}+1\right)\left(v_{1}^{2}+v_{2}^{2}\right)+4 n v_{1} v_{2} \geq\left\{\begin{array}{cl}
(n-1)^{2}\left(v_{1}^{2}+v_{2}^{2}\right) & \text { if } n \neq 1 \\
2\left(v_{1}+v_{2}\right)^{2} & \text { if } n=1
\end{array}\right.
$$

and the following Poincaré inequality

$$
\iint_{\Omega}\left(\sum_{i=1}^{3}\left|\frac{\partial v_{i}}{\partial z}\right|^{2}\right) r \mathrm{~d} r \mathrm{~d} z \geq \frac{\pi^{2}}{4 h^{2}} \iint_{\Omega}|v|^{2} r \mathrm{~d} r \mathrm{~d} z, \text { for } v \in V_{n}
$$

Hence, we have proved the following

Proposition 3.1. The bilinear form $a_{n}(u, v)$ is coercive i.e. there exists a constant $K>0$ (independent of $n$ ) such that

$$
a_{n}(u, u) \geq K\|u\|_{V_{n}}^{2}, \quad \forall u \in V_{n}
$$

Applying the Kato first representation theorem (see [7]), we assert that $a_{n}(.,$.$) spans a self-adjoint operator A_{n}$ defined in $H$ by:

$$
\left\{\begin{aligned}
D\left(A_{n}\right) & =\left\{u \in V_{n} ; \mathcal{A}_{n} u \in H \text { and } \sigma_{r z}^{n}(u)(r, h)=\sigma_{\theta z}^{n}(u)(r, h)=\sigma_{z z}^{n}(u)(r, h)=0\right\}, \\
A_{n} u & =\mathcal{A}_{n} u \text { for } u \in D\left(A_{n}\right) .
\end{aligned}\right.
$$

Using the generalized Green's formula, we obtain

$$
a_{n}(u, v)=\left(A_{n} u, v\right) \quad \text { for }(u, v) \in D\left(A_{n}\right) \times V_{n} .
$$

Notice that the traces $\sigma_{\tau z}^{n}(u)(r, h), \tau=r, \theta, z$, lie in the space $H_{l o c}^{-1 / 2}\left(\mathbb{R}^{+}\right)$.

Now we are going to study the spectrum of the operator $A_{n}$, but in a preliminary step we study the essential spectrum. First of all we prove a result on the regularity of an element $u \in D\left(A_{n}\right)$ in the exterior domain $\Omega^{\prime} R_{1}=$ ]$R_{1},+\infty[\times] 0, h\left[\right.$ with $R_{1}>R$ (i.e. outside the perturbation). Such a result will be useful in Paragraph 5.1 to get a compactness result.

\section{A Regularity Result}

We consider the equation:

$$
A_{n} u=\tilde{f}, \quad(\tilde{f} \in H)
$$


Due to the coerciveness of $a_{n}(.,$.$) , the estimation \|u\|_{V_{n}} \leq C\|\tilde{f}\|_{H}$ follows. Our purpose now is to obtain a better estimation on $\Omega^{\prime} R_{1}$, namely:

$$
\|\sqrt{r} u\|_{2, \Omega^{\prime} R_{1}} \leq C\left(\|\tilde{f}\|_{H}+\|u\|_{V_{n}}\right)
$$

This inequality would be obvious if the domain was bounded. First of all we transform the equation (22). We set $v=\sqrt{r} u$, then $v$ satisfies in $\left.\Omega^{\prime}{ }_{R}=\right] R,+\infty[\times] 0, h[$ the system:

$$
\left\{\begin{array}{l}
\mu_{\infty}\left(\frac{\partial^{2} v_{1}}{\partial r^{2}}+\frac{\partial^{2} v_{1}}{\partial z^{2}}\right)+\left(\lambda_{\infty}+\mu_{\infty}\right) \frac{\partial}{\partial r}\left(\frac{\partial v_{1}}{\partial r}+\frac{\partial v_{3}}{\partial z}\right)=f_{1}(v) \\
\frac{\partial^{2} v_{2}}{\partial r^{2}}+\frac{\partial^{2} v_{2}}{\partial z^{2}}=f_{2}(v) \\
\mu_{\infty}\left(\frac{\partial^{2} v_{3}}{\partial r^{2}}+\frac{\partial^{2} v_{3}}{\partial z^{2}}\right)+\left(\lambda_{\infty}+\mu_{\infty}\right) \frac{\partial}{\partial z}\left(\frac{\partial v_{1}}{\partial r}+\frac{\partial v_{3}}{\partial z}\right)=f_{3}(v)
\end{array}\right.
$$

where $f(v)=\left(f_{1}(v), f_{2}(v), f_{3}(v)\right)$ satisfies the inequality:

$$
\|f(v)\|_{0, \Omega_{R}^{\prime}} \leq c\left(\|\tilde{f}\|_{H}+\|u\|_{V_{n}}\right)
$$

For $\bar{v}=\left(v_{1}, v_{3}\right)$, we consider the quantities:

$$
\left\{\begin{array}{l}
\sigma_{13}(\bar{v})=\mu_{\infty}\left(\frac{\partial v_{3}}{\partial r}+\frac{\partial v_{1}}{\partial z}\right) \\
\sigma_{33}(\bar{v})=\left(\lambda_{\infty}+2 \mu_{\infty}\right) \frac{\partial v_{3}}{\partial z}+\lambda_{\infty} \frac{\partial v_{1}}{\partial r}
\end{array}\right.
$$

The condition of free surface $\left\{\sigma_{r z}^{n}(u)=\sigma_{\theta z}^{n}(u)=\sigma_{z z}^{n}(u)=0\right.$, for $\left.z=h\right\}$ implies together with (6) that

$$
\left\{\begin{array}{l}
\sigma_{13}(\bar{v})+\mu_{\infty} \frac{u_{3}}{2 \sqrt{r}}=0, \quad \text { for } z=h \\
\sigma_{33}(\bar{v})+\lambda_{\infty}\left(\frac{u_{1}}{2 r}+\frac{u_{1}+n u_{2}}{r \sqrt{r}}\right)=0, \quad \text { for } z=h \\
\frac{\partial v_{2}}{\partial z}(r, h)+\frac{n}{r \sqrt{r}} u_{2}(r, h)=0
\end{array}\right.
$$

Consequently, the traces $\sigma_{13}(\bar{v})(r, h), \sigma_{33}(\bar{v})(r, h)$ and $\frac{\partial v_{2}}{\partial z}(r, h)$ lie in the space $H^{1 / 2}(R,+\infty)$. Then the field $\bar{v} \in\left[H^{1}\left(\Omega_{R}^{\prime}\right)\right]^{2}$ satisfies the $2 \mathrm{D}$ elasticity problem:

$$
\left\{\begin{array}{l}
A \bar{v}=f \quad \text { in } \Omega_{R}^{\prime}, \\
\bar{v}(r, 0)=0, \quad \forall r>R, \\
\sigma_{13}(\bar{v})(r, h)=g_{1}(r), \sigma_{33}(\bar{v})(r, h)=g_{3}(r), \quad \forall r>R,
\end{array}\right.
$$

with

$$
A \bar{v}=\mu_{\infty} \Delta \bar{v}+\left(\lambda_{\infty}+\mu_{\infty}\right) \nabla(\nabla \cdot \bar{v})
$$

Moreover we have:

$$
\left.\left\|g_{1}\right\|_{\frac{1}{2}}+\left\|g_{3}\right\|_{\frac{1}{2}} \leq c\|u\|_{V_{n}} \quad \text { (with }\|g\|_{s}:=\|g\|_{H^{s}}\right)
$$


The function $v_{2} \in H^{1}\left(\Omega_{R}^{\prime}\right)$ satisfies the scalar problem:

$$
\left\{\begin{array}{l}
\Delta v_{2}=f_{2}, \quad \text { in } \Omega_{R}^{\prime}, \\
v_{2}(r, 0)=0, \\
\frac{\partial v_{2}}{\partial z}(r, h)=g_{2}(r), \quad \forall r>R,
\end{array}\right.
$$

with the estimation $\left\|g_{2}\right\|_{\frac{1}{2}} \leq c\|u\|_{V_{n}}$.

Now we look at the regularity of $v$ in the unbounded domain $\Omega^{\prime} R_{1}$. Without loss of generality, we can suppose that $R_{1}=R+2$, then there exists a cut-off function $\varphi \in D(]-1,1[)$ such that:

$$
\sum_{m \geq 0} \varphi(r-m)=1, \quad \forall r>0 .
$$

We let $\left.\varphi_{m}(r)=\varphi(r-m), I_{m}=\right] m-1, m+1\left[\right.$ and $\left.\Omega_{m}=I_{m} \times\right] 0, h[$.

Setting $v_{m}(r, z)=\varphi_{m}(r) v(r, z)$ where $v$ is the solution of $\left(P_{1}\right)-\left(P_{2}\right)$, we see that $\bar{v}_{m}=\left(v_{m 1}, v_{m 3}\right)$ and $v_{m 2}$ satisfy regular elliptic boundary-value problems in the bounded domain $\Omega_{m}$. Hence $v_{m} \in H^{2}\left(\Omega_{m}\right)^{3}$ and we have the following inequality:

$$
\left\|v_{m}\right\|_{2, \Omega_{m}} \leq c\left(\|f\|_{0, \Omega}+\|g\|_{\frac{1}{2}}\right),
$$

where the constant $c$ is independent of $m$ (the diameter of $\Omega_{m}$ which is uniformly bounded). Since $v=\sum_{m \geq m_{0}} v_{m}$ on $\Omega^{\prime} R_{1}$ where $m_{0}$ is the integer part of $R_{1}$, we obtain:

$$
\|v\|_{2, \Omega} \leq c_{1} \sum_{m \geq m_{0}}\left\|v_{m}\right\|_{2, \Omega_{m}} \leq c_{2}\left(\|f\|_{0, \Omega}+\|g\|_{\frac{1}{2}}\right) .
$$

Hence we have the result:

Proposition 4.1. Let $u \in V_{n}$ be a solution of (22), then $u$ satisfies:

(1) $\sqrt{r} u \in H^{2}\left(\Omega_{R_{1}}^{\prime}\right)^{3}$ with $\left.\Omega_{R_{1}}^{\prime}=\right] R_{1},+\infty[\times] 0, h\left[\right.$ and $R_{1}>R$,

(2) $\|\sqrt{r} u\|_{2, \Omega_{R_{1}}^{\prime}} \leq c\left(R_{1}\right)\left(\|\tilde{f}\|_{H}+\|u\|_{V_{n}}\right)$.

\section{The essential SpeCtrum of $A_{n}$}

The operator $A_{n}$ is self-adjoint and non negative, then its spectrum is a subset of $\mathbb{R}_{+}$. Since the imbedding of $V_{n}$ in $H$ is not compact, the resolvent of $A_{n}$ is not compact and its spectrum $\sigma\left(A_{n}\right)$ is composed of a continuous part (the essential spectrum $\left.\sigma_{e}\left(A_{n}\right)\right)$ and sometimes of a discrete part $\sigma_{d}\left(A_{n}\right)$ corresponding to the eigenmodes. We recall (see [12]) that a real number $\sigma \in \sigma_{e}\left(A_{n}\right)$ if and only if there exists a singular sequence $u^{p} \in D\left(A_{n}\right)$ such that:

$$
\left\|u^{p}\right\|=1, u^{p} \rightarrow 0 \text { weakly in } H \quad \text { and } A_{n} u^{p}-\sigma u^{p} \rightarrow 0 \text { in } H .
$$

Often the essential spectrum of the global operator $A$, defined on $\tilde{\Omega}$ as Hilbertian sum of the operators $A_{n}$, coincides with the spectrum of the non-perturbed operator $\bar{A}$ (corresponding to the homogeneous layer). The study of the reduced operators (obtained after performing Fourier transformation in the horizontal direction) enables us to predict the lower bound of $\sigma(\bar{A})$. Thus we should have $\sigma_{e}\left(A_{n}\right) \subset \sigma(\bar{A})$. But the initial hypothesis $\left(\sigma_{e}(A)=\sigma(\bar{A})\right)$ is not proved in this general case. We can prove this strictly (as in [5]) under a restrictive 
hypothesis on Lamé coefficients, namely if $\lambda$ and $\mu$ are Lipschitz continuous functions, which restricts the generality. The aim of this section is to prove the inclusion $\sigma_{e}\left(A_{n}\right) \subset \sigma(\bar{A})$ in the general case (i.e. when $\left.\lambda, \mu \in L^{\infty}(\Omega)\right)$. First of all we establish a compactness result based on Proposition 4.1.

\subsection{A compactness result}

We recall some properties of weighted Sobolev spaces. To our knowledge theses spaces have not been used before on the similar works in waveguides.

Definitions. For $s, t \in \mathbb{R}$, we define the Hilbert spaces:

$$
L^{2, s}(\mathbb{R})=\left\{u \in L_{\text {loc }}^{2}(\mathbb{R}) ; \quad \rho^{s} u \in L^{2}(\mathbb{R})\right\}, \quad H^{s, t}(\mathbb{R})=\left\{u \in S^{\prime}(\mathbb{R}) ; \quad F\left(\rho^{t} u\right) \in L^{2, s}(\mathbb{R})\right\}
$$

with the norms $\|u\|_{s, t}=\left\|F\left(\rho^{t} u\right)\right\|_{L_{s}^{2}}$ where $F$ is the Fourier transform and $\rho(x)=\left(1+x^{2}\right)^{1 / 2}$.

If $I \subset \mathbb{R}$ is an unbounded interval we set $H^{s, t}(I)=\left\{u=\left.v\right|_{I} ; v \in H^{s, t}(\mathbb{R})\right\}$.

We have the properties:

1) If $s \geq s^{\prime}$ and $t \geq t^{\prime}$ then the embedding $H^{s, t}(\mathbb{R}) \subset H^{s^{\prime}, t^{\prime}}(\mathbb{R})$ is continuous.

2) If $s>s^{\prime} \geq 0$ and $t>t^{\prime}$ then the embedding $H^{s, t}(\mathbb{R}) \subset H^{s^{\prime}, t^{\prime}}(\mathbb{R})$ is compact.

3) If $s \geq 0$ and $t \in \mathbb{R}$ then the dual $\left(H^{s, t}(\mathbb{R})\right)^{\prime}=H^{-s,-t}(\mathbb{R})$.

Proposition 5.1. Let $\left(u^{p}\right)_{p \in \mathbb{N}}$ be a sequence of $D\left(A_{n}\right)$ satisfying $\left\|A_{n} u^{p}\right\|_{H} \leq c$ for all $p$. Then we can extract a subsequence, still denoted $\left(u^{p}\right)$, which converges weakly in $V_{n}$ to an element $u$ such that:

(i) $\sqrt{r} u^{p}(., h) \rightarrow \sqrt{r} u(., h)$ strongly in $Y^{*}=\left(H^{-\frac{1}{2}, t}(\bar{R},+\infty)\right)^{3}$ with $\bar{R}>R$ and $t>0$,

(ii) $\sqrt{r} \frac{\partial u^{p}}{\partial r}(., h) \rightarrow \sqrt{r} \frac{\partial u}{\partial r}(., h)$ weakly in $Y=\left(H^{\frac{1}{2},-t}(\bar{R},+\infty)\right)^{3}$,

(iii) $u^{p}(., h) \rightarrow u(., h)$ strongly in $\left(L^{2}(\bar{R},+\infty)\right)^{3}$.

Proof. It follows from the coerciveness of $a_{n}(.,$.$) that \left\|u^{p}\right\|_{V_{n}} \leq c$ for all $p$. Then there exists a subsequence which converges weakly in $V_{n}$ to an element $u$. The proposition 4.1 shows that $\forall p, \quad\left\|\sqrt{r} u^{p}\right\|_{2, \Omega_{\bar{R}}^{\prime}} \leq c\left(\left\|A_{n} u^{p}\right\|_{H}+\right.$ $\left.\left\|u^{p}\right\|_{V_{n}}\right) \leq c_{1}$. This implies that the sequence of traces $\left(\sqrt{r} u^{p}(., h)\right)_{p}$ is bounded in the space $H^{\frac{3}{2}}(\bar{R},+\infty)^{3}$. This last space is compactly embedded in the space $Y=H^{\frac{1}{2},-t}(\bar{R},+\infty)^{3}$ with $t>0$ arbitrary. Therefore $\sqrt{r} u^{p}(., h)$ tends to $\sqrt{r} u(., h)$ strongly in $Y$. Since the embedding $Y \subset Y^{*}=H^{-\frac{1}{2}, t}(\bar{R},+\infty)^{3}$ is continuous, we obtain $(i)$.

The sequence $\sqrt{r} \frac{\partial u^{p}}{\partial r}(r, h)$ is bounded in $H^{\frac{1}{2}}(\bar{R},+\infty)^{3}$, hence in $Y$, which proves the property (ii). The property (iii) follows from the fact that the sequence $\left(u^{p}(., h)\right)$ is bounded in $H^{\frac{1}{2}}, \frac{1}{2}(\bar{R},+\infty)^{3}$ which is embedded compactly in $L^{2}(\bar{R},+\infty)^{3}$.

Lemma 5.2. For every $u \in D\left(A_{n}\right)$ :

$$
a_{n}(u, u) \geq \mu_{\infty} \iint_{\Omega_{\bar{R}}^{\prime}}|\nabla u|^{2} r \mathrm{~d} r \mathrm{~d} z+\mu_{\infty} p_{n}(u, u), \quad(\bar{R}>R),
$$

with

$$
\begin{aligned}
p_{n}(u, u)= & 2 \int_{\bar{R}}^{+\infty}\left[r \frac{\partial u_{3}}{\partial r}(r, h) u_{1}(r, h)-n u_{2}(r, h) u_{3}(r, h)\right] \mathrm{d} r \\
& +\int_{0}^{h}\left[u_{1}^{2}(\bar{R}, z)+u_{2}^{2}(\bar{R}, z)+2 n u_{1}(\bar{R}, z) u_{2}(\bar{R}, z)\right] \mathrm{d} z \\
& -2 \int_{0}^{h} \bar{R} \frac{\partial u_{3}}{\partial z}(\bar{R}, z) u_{1}(\bar{R}, z) \mathrm{d} z
\end{aligned}
$$


Proof. $a_{n}(.,$.$) can be written in the form:$

$$
a_{n}(u, u)=\iint_{\Omega} \lambda\left|\operatorname{div}^{n} u\right|^{2} r \mathrm{~d} r \mathrm{~d} z+\iint_{\Omega} \mu \varepsilon_{n}(u, u) \mathrm{d} r \mathrm{~d} z
$$

with $\operatorname{div}^{n} u$ given in (12) and

$$
\begin{aligned}
r \varepsilon_{n}(u, u)= & r\left|\operatorname{div}^{n} u\right|^{2}+r|\nabla u|^{2}+\frac{1}{r}\left(\left(n^{2}+1\right)\left(u_{1}^{2}+u_{2}^{2}\right)+n^{2} u_{3}^{2}+4 n u_{1} u_{2}\right) \\
& +2 r\left(\frac{\partial u_{1}}{\partial z} \frac{\partial u_{3}}{\partial r}-\frac{\partial u_{1}}{\partial r} \frac{\partial u_{3}}{\partial z}\right)-2\left(n u_{1}+u_{2}\right) \frac{\partial u_{2}}{\partial r} \\
& -2\left(u_{1}+n u_{2}\right) \frac{\partial u_{1}}{\partial r}-2 n \frac{\partial}{\partial z}\left(u_{2} u_{3}\right)-2 u_{1} \frac{\partial u_{3}}{\partial z} .
\end{aligned}
$$

Using the inequality (20) the decomposition (25) gives

$$
\begin{aligned}
a_{n}(u, u) \geq & \mu_{\infty} \iint_{\Omega_{\bar{R}}^{\prime}}|\nabla u|^{2} r \mathrm{~d} r \mathrm{~d} z \\
& +2 \mu_{\infty} \iint_{\Omega_{\bar{R}}^{\prime}}\left[r\left(\frac{\partial u_{1}}{\partial z} \frac{\partial u_{3}}{\partial r}-\frac{\partial u_{1}}{\partial r} \frac{\partial u_{3}}{\partial z}\right)-\left(n u_{1}+u_{2}\right) \frac{\partial u_{2}}{\partial r}\right] \mathrm{d} r \mathrm{~d} z \\
& -2 \mu_{\infty} \iint_{\Omega_{\bar{R}}^{\prime}}\left[\left(u_{1}+n u_{2}\right) \frac{\partial u_{1}}{\partial r}+n \frac{\partial}{\partial z}\left(u_{2} u_{3}\right)+u_{1} \frac{\partial u_{3}}{\partial z}\right] \mathrm{d} r \mathrm{~d} z .
\end{aligned}
$$

Since $\sqrt{r} u \in H^{2}(] \bar{R},+\infty[\times] 0, h[)^{3}$ (see Prop. 4.1), we integrate twice by parts to get (24).

The following proposition is the key result which will be useful in the next paragraph to determine the lower bound of the essential spectrum of $A_{n}$.

Proposition 5.3. Let $\left(u_{p}\right)$ be a singular sequence of $D\left(A_{n}\right)$. Then

$$
\lim _{p \rightarrow+\infty} p_{n}\left(u^{p}, u^{p}\right)=0
$$

Proof. It follows from the properties of singular sequence (see (23)) that $u^{p} \rightarrow 0$ weakly in $V_{n}$ and the sequence $\left(A_{n} u^{p}\right)_{p}$ is bounded in $H$. The result follows from the regularity of $u^{p}$ (see Prop. 4.1) and the compactness result (see Prop. 5.1) noting that when $p \rightarrow+\infty$

$$
\int_{\bar{R}}^{+\infty} u_{1}^{p}(r, h) \frac{\partial u_{3}^{p}}{\partial r}(r, h) r \mathrm{~d} r=\left\langle\sqrt{r} \frac{\partial u_{3}^{p}}{\partial r}(r, h), \sqrt{r} u_{1}^{p}(r, h)\right\rangle_{Y \times Y^{*}} \rightarrow 0 .
$$

\subsection{The essential spectrum}

The following lemma gives a lower bound for the essential spectrum of $A_{n}$.

Lemma 5.4. For all $n \in \mathbb{N}$ we have:

$$
\sigma_{e}\left(A_{n}\right) \subset\left[\omega_{1}^{2},+\infty\left[\quad \text { with } \omega_{1}^{2}=\frac{\pi^{2}}{4 h^{2}} c_{S}^{2}\right.\right.
$$


Proof. Let $\omega^{2} \in \sigma_{e}\left(A_{n}\right)$. We suppose the contrary (i.e. $\left.\omega^{2}<\omega_{1}^{2}\right)$ and let $\left(u_{p}\right)$ the associated singular sequence. The sequence $\left(u^{p}\right)$ is bounded in $\left.V_{n}\left(\Omega_{\bar{R}}\right)=\left\{u=\left.v\right|_{\Omega_{\bar{R}}}, v \in V_{n}\right\}, \Omega_{\bar{R}}=\right] 0, \bar{R}[\times] 0, h[$, which is embedded compactly in $L^{2}\left(\Omega_{\bar{R}}\right)$. Then we can extract a subsequence satisfying

$$
\lim _{p \rightarrow \infty} \iint_{\Omega_{\bar{R}}}\left|u^{p}\right|^{2} \rho r \mathrm{~d} r \mathrm{~d} z=0 .
$$

Combining Lemma 5.2 and Poincaré inequality (21) we obtain the inequality

$$
a_{n}\left(u^{p}, u^{p}\right) \geq \omega_{1}^{2} \iint_{\Omega_{\bar{R}}^{\prime}}\left|u^{p}\right|^{2} \rho_{\infty} r \mathrm{~d} r \mathrm{~d} z+\mu_{\infty} p_{n}\left(u^{p}, u^{p}\right)
$$

therefore

$$
a_{n}\left(u^{p}, u^{p}\right)-\omega^{2} \geq\left(\omega_{1}^{2}-\omega^{2}\right) \iint_{\Omega_{\bar{R}}^{\prime}}\left|u^{p}\right|^{2} \rho_{\infty} r \mathrm{~d} r \mathrm{~d} z-\omega^{2} \iint_{\Omega_{\bar{R}}}\left|u^{p}\right|^{2} \rho r \mathrm{~d} r \mathrm{~d} z+\mu_{\infty} p_{n}\left(u^{p}, u^{p}\right) .
$$

As $\lim _{p \rightarrow \infty} a_{n}\left(u^{p}, u^{p}\right)=\omega^{2}$, the Proposition 5.3 together with (27) and (28) lead to

$$
\lim _{p \rightarrow \infty} \iint_{\Omega_{\bar{R}}^{\prime}}\left|u^{p}\right|^{2} \rho_{\infty} r \mathrm{~d} r \mathrm{~d} z=0
$$

which is in contradiction with $\iint_{\Omega}\left|u^{p}\right|^{2} \rho r \mathrm{~d} r \mathrm{~d} z=1$, which shows $\omega^{2} \geq \omega_{1}^{2}$.

Theorem 5.5. For all $n \in \mathbb{N}$ we have $\sigma_{e}\left(A_{n}\right)=\left[\omega_{1}^{2},+\infty[\right.$.

Proof. We will now construct singular sequences to prove that the inclusion (26) is in fact an equality. For $\sigma>\omega_{1}^{2}$, we built a generalized eigenfunction $u^{\sigma}$ of the non-perturbed operator $\bar{A}_{n}$, obtained from $A_{n}$ by putting $(\lambda, \mu, \rho)=\left(\lambda_{\infty}, \mu_{\infty}, \rho_{\infty}\right)$. To do this, let us consider the following problem:

$$
\text { Find } u^{\sigma} \in D_{l o c}\left(\bar{A}_{n}\right) \cap L^{\infty}(\Omega)^{3} \text { such that } \bar{A}_{n} u^{\sigma}=\sigma u^{\sigma} \text {. }
$$

This problem can be solved explicitly by using the method of potentials (see for example [8]). The field $u^{\sigma}$ is given by

$$
\left\{\begin{array}{l}
u_{1}^{\sigma}(r, z)=\left(J_{n-1}(k r)-\frac{n}{r} J_{n}(k r)\right)\left(k g_{1}(z)+g_{2}^{\prime}(z)\right) \\
u_{2}^{\sigma}(r, z)=-\frac{n}{r} J_{n}(k r)\left(g_{1}(z)+\frac{1}{k} g_{2}^{\prime}(z)\right) \\
u_{3}^{\sigma}(r, z)=J_{n}(k r)\left(g_{1}(z)+k g_{2}^{\prime}(z)\right)
\end{array}\right.
$$

where $J_{n}(r)$ is the Bessel's function of the first kind and of order $n$, and

$$
\left\{\begin{array}{l}
g_{1}(z)=A(k) \cos (\alpha z)+B(k) \sin (\alpha z) \\
g_{2}(z)=C(k) \cos (\beta z)+D(k) \sin (\beta z)
\end{array}\right.
$$

with $\alpha^{2}=\frac{\sigma}{c_{P}^{2}}-k^{2}, \beta^{2}=\frac{\sigma}{c_{S}^{2}}-k^{2}$ and $k=k_{1}(\sigma)$ is an analytic function defined implicitly by the following dispersion equation:

$2 k^{2} \alpha \beta\left[\xi-\mu_{\infty}\left(k^{2}-\beta^{2}\right)\right]+\alpha \beta\left[4 k^{2} \mu_{\infty}-\xi\left(k^{2}-\beta^{2}\right] \cos (\alpha h) \cos (\beta h)+\right.$

$$
k^{2}\left[\xi\left(k^{2}-\beta^{2}\right)-4 \mu_{\infty} \alpha^{2} \beta^{2}\right] \sin (\alpha h) \sin (\beta h)=0
$$


with $\xi=\lambda_{\infty} \frac{\sigma}{c_{P}^{2}}+2 \mu_{\infty} \alpha^{2}$. The curve $\sigma \rightarrow k_{1}(\sigma), \sigma>\omega_{1}^{2}$, is plotted numerically, it is strictly increasing and has the properties: $k_{1}(\sigma)>0$ if $\sigma>\omega_{1}^{2}$ and $\lim _{\sigma \rightarrow \infty} \frac{\sigma}{k_{1}(\sigma)}=c_{P}^{2}$.

The field $u^{\sigma}$ is not in $V_{n}$. Consider now the sequence $\left(u_{p}\right)$ in $H$ defined as follows:

$$
u_{p}(r, z)=\frac{1}{\sqrt{p}} \varphi_{p}(r) u^{\sigma}(r, z)
$$

where $\left(\varphi_{p}\right)$ is a sequence of cut-off functions defined by:

$$
\left\{\begin{array}{l}
\varphi_{p}(r)=\varphi\left(\frac{r}{p}\right), \\
\varphi \in C_{0}^{\infty}(] 0,+\infty[), \varphi(r)=0 \text { if } r<R, \\
\int_{0}^{+\infty}|\varphi|^{2} \mathrm{~d} r=1 .
\end{array}\right.
$$

The sequence $\left(u^{p}\right)$ satisfies all the properties of a singular sequence. This proves that $\sigma \in \sigma_{e}\left(A_{n}\right)$. As $\sigma_{e}\left(A_{n}\right)$ is a closed set, the theorem is proved.

\section{The Point SPECTRUM}

We are interested now in the point spectrum of the operator $A_{n}$. The eigenvalues $\omega^{2}$ such that $\omega^{2}<\omega_{1}^{2}$ form the discrete spectrum; they can be studied by means of the Min-Max principle (see [11]) as will be seen in the following. The study of the so-called embedded eigenvalues such that $\omega^{2} \geq \omega_{1}^{2}$ is much more complicated. Obviously, this discrete spectrum may be empty: it is the case for example if the layer is homogeneous. Therefore, our goal is to find out the types of perturbations for which eigenmodes exist.

Hypothesis. Suppose that there exists a rectangle $G=] a, b[\times] 0, h[, a \geq 0$, such that:

$$
\mu(r, z)=\mu_{\infty} \quad \text { and } \quad \rho(r, z)=\rho_{\infty} \quad \text { in } \Omega \backslash G .
$$

We put: $\mu_{0}=\sup _{G} \mu(r, z)$ and $\lambda_{0}=\sup _{G} \lambda(r, z)$.

Theorem 6.1. Suppose that the couple $(\mu, \rho)$ satisfies hypothesis (H1), then there exists a number $\delta>0$ (which depends of $\left.\left(n, h, b-a, \mu_{0}, \lambda_{0}\right)\right)$ such that the discrete spectrum of $A_{n}$ is non empty if

$$
\mu_{\infty}-\mu_{0} \geq \delta
$$

Proof. We consider the Bessel's operator

$$
\left\{\begin{array}{l}
\left.B_{1} v=-\frac{1}{r} \frac{\mathrm{d}}{\mathrm{d} r}\left(r \frac{\mathrm{d} v}{\mathrm{~d} r}\right)+\frac{v}{r^{2}} \quad \text { in } I=\right] a, b[, \\
v(a)=v(b)=0 .
\end{array}\right.
$$

Denoting by $v$ the eigenfunction of $B_{0}$ associated to the first eigenvalue $\alpha_{1}$, we have:

$$
\int_{I}\left(r\left|\frac{\mathrm{d} v}{\mathrm{~d} r}\right|^{2}+\frac{v^{2}}{r}\right) \mathrm{d} r=\alpha_{1} \quad \text { and } \int_{I}|v|^{2} r \mathrm{~d} r=1 .
$$


Considering the function $g(z)=\sqrt{\frac{2}{\rho_{\infty} h}} \sin \left(\frac{\omega_{1}}{c_{S}} z\right)$, we can verify that:

$$
\int_{0}^{h}|g|^{2} \rho_{\infty} \mathrm{d} z=1 \text { and } \int_{0}^{h}\left|g^{\prime}\right|^{2} \mu_{\infty} \mathrm{d} z=\omega_{1}^{2} .
$$

Now we put $u(r, z)=(w(r) g(z), 0,0)$, where $w(r)$ is the extension by zero of $v(r)$ outside the interval $I$. Then we have:

$$
\begin{aligned}
a_{n}(u, u)= & \iint_{\Omega}\left[(\lambda+2 \mu) r\left|\frac{\mathrm{d} w}{\mathrm{~d} r}\right|^{2}|g|^{2}+2 \lambda w \frac{\mathrm{d} w}{\mathrm{~d} r}|g|^{2}\right. \\
& \left.+\frac{\lambda+\left(n^{2}+2\right) \mu}{r}|w|^{2}|g|^{2}+r \mu|w|^{2}\left|\frac{\mathrm{d} g}{\mathrm{~d} z}\right|^{2}\right] \mathrm{d} r \mathrm{~d} z \\
= & \iint_{\Omega}(\lambda+2 \mu) r\left(\frac{\mathrm{d} w}{\mathrm{~d} r}+\frac{w}{r}\right)^{2}|g|^{2} \mathrm{~d} r \mathrm{~d} z-4 \iint_{\Omega} \mu w \frac{\mathrm{d} w}{\mathrm{~d} r}|g|^{2} \mathrm{~d} r \mathrm{~d} z \\
& +\iint_{\Omega} r \mu|w|^{2}\left|\frac{\mathrm{d} g}{\mathrm{~d} z}\right|^{2} \mathrm{~d} r \mathrm{~d} z+n^{2} \iint_{\Omega} \frac{\mu}{r}|w|^{2}|g|^{2} \mathrm{~d} r \mathrm{~d} z .
\end{aligned}
$$

On the other hand we have:

$$
\begin{aligned}
\iint_{\Omega}(\lambda+2 \mu) r\left(\frac{\mathrm{d} w}{\mathrm{~d} r}+\frac{w}{r}\right)^{2}|g|^{2} \mathrm{~d} r \mathrm{~d} z & \leq \frac{\lambda_{0}+2 \mu_{0}}{\rho_{\infty}} \int_{I}\left(\frac{\mathrm{d} v}{\mathrm{~d} r}+\frac{v}{r}\right)^{2} r \mathrm{~d} r \times \int_{0}^{h}|g|^{2} \rho_{\infty} \mathrm{d} z \\
& \leq \frac{\lambda_{0}+2 \mu_{0}}{\rho_{\infty}} \alpha_{1} \\
\iint_{\Omega} \frac{\mu}{r}|w|^{2}|g|^{2} \mathrm{~d} r \mathrm{~d} z & \leq \frac{\mu_{0}}{\rho_{\infty}} \int_{I}|v|^{2} \frac{\mathrm{d} r}{r} \leq \frac{\mu_{0}}{\rho_{\infty}} \alpha_{1},
\end{aligned}
$$

and according to $(H 2)$ we have:

$$
\iint_{\Omega} r \mu|w|^{2}\left|\frac{\mathrm{d} g}{\mathrm{~d} z}\right|^{2} \mathrm{~d} r \mathrm{~d} z=\iint_{G} r \mu|v|^{2}\left|\frac{\mathrm{d} g}{\mathrm{~d} z}\right|^{2} \mathrm{~d} r \mathrm{~d} z \leq \int_{0}^{h}\left(\mu_{\infty}-\delta\right)\left|\frac{\mathrm{d} g}{\mathrm{~d} z}\right|^{2} \mathrm{~d} z \leq \omega_{1}^{2}-\delta \frac{\omega_{1}^{2}}{\mu_{\infty}} .
$$

We also have

$$
4 \iint_{\Omega} \mu|w|\left|\frac{\mathrm{d} w}{\mathrm{~d} r}\right||g|^{2} \mathrm{~d} r \mathrm{~d} z \leq 4 \frac{\mu_{0}}{\rho_{\infty}} \int_{I}|v|\left|\frac{\mathrm{d} v}{\mathrm{~d} r}\right| \mathrm{d} r \leq 2 \frac{\mu_{0}}{\rho_{\infty}} \int_{I}\left(\frac{|v|^{2}}{r}+r\left|\frac{\mathrm{d} v}{\mathrm{~d} r}\right|^{2}\right) \mathrm{d} r=2 \alpha_{1} \frac{\mu_{0}}{\rho_{\infty}}
$$

which gives

$$
a_{n}(u, u)-\omega_{1}^{2}\|u\|^{2} \leq \alpha_{1}\left(\frac{\lambda_{0}+\left(n^{2}+4\right) \mu_{0}}{\rho_{\infty}}\right)-\delta \frac{\omega_{1}^{2}}{\mu_{\infty}}
$$

The left hand side is negative if $\delta>\frac{4 \alpha_{1} h^{2}}{\pi^{2}}\left[\lambda_{0}+\left(n^{2}+4\right) \mu_{0}\right]$. The Min-Max principle proves that $A_{n}$ has at least one eigenvalue less than $\omega_{1}^{2}$.

\section{Remark 6.2.}

1) If $a=0$ then $\alpha_{1}=\left(\frac{\beta_{1}}{b}\right)^{2}$ where $\beta_{1}$ is the first root of Bessel function $J_{1}(r)$. More generally $\alpha_{1}$ decreases when $(b-a)$ grows.

2) Theorem 6.1 means that if in an elastic layer there is a region which is considerably softer than the rest then one would expect a "trapped mode" localised around the soft region, moreover it is a radial field. From the proof we can see that only a finite number of harmonics occur in the layer. This number increases with $\delta_{0}=\mu_{\infty}-\mu_{0}$. 
The following theorem complete the previous one.

Theorem 6.3. If the following condition is satisfied:

$$
\iint_{\Omega_{R}}\left(\frac{\mu}{\rho}\left|g^{\prime}\right|^{2}-\omega_{1}^{2}|g|^{2}\right) \rho r \mathrm{~d} r \mathrm{~d} z<0, \quad \text { with } g(z)=\sqrt{\frac{2}{\rho_{\infty} h}} \sin \left(\frac{\omega_{1}}{c_{S}} z\right),
$$

then the operator $A_{1}$ has at least one discrete eigenvalue.

Proof. It suffices to find $u \in V_{1}$ such that $a_{1}(u, u)-\omega_{1}^{2}\|u\|^{2}<0$. For $a>R$ we define $\varphi_{a}(r)$ by:

$$
\varphi_{a}(r)=\left\{\begin{array}{cl}
1 & \text { if } 0 \leq r \leq R \\
\frac{\log \frac{a}{r}}{\log \frac{a}{R}} & \text { if } R \leq r \leq a \\
0 & \text { if } a \leq r
\end{array}\right.
$$

We verify that: $\sqrt{r} \varphi_{a} \in L^{2}\left(\mathbb{R}^{+}\right), \quad \sqrt{r} \varphi_{a}^{\prime} \in L^{2}\left(\mathbb{R}^{+}\right)$and $\lim _{a \rightarrow \infty} \int_{0}^{\infty}\left|\varphi_{a}^{\prime}\right|^{2} r \mathrm{~d} r=0$.

Putting $u(r, z)=\left(\varphi_{a}(r) g(z),-\varphi_{a}(r) g(z), 0\right)$, then $u \in V_{1}$ and the decomposition (25) gives for $n=1$ :

$$
a_{1}(u, u)-\omega_{1}^{2}\|u\|^{2}=b_{1}(u, u)
$$

with

$$
\begin{aligned}
b_{1}(u, u) & =\iint_{\Omega}\left((\lambda+3 \mu)\left|\varphi_{a}^{\prime}\right|^{2}|g|^{2}+2 \mu\left|\varphi_{a}\right|^{2}\left|g^{\prime}\right|^{2}-\omega_{1}^{2}|g|^{2}\left|\varphi_{a}\right|^{2} \rho\right) r \mathrm{~d} r \mathrm{~d} z \\
& =\left(\lambda_{\infty}+3 \mu_{\infty}\right) \int_{0}^{\infty}\left|\varphi_{a}^{\prime}\right|^{2} r \mathrm{~d} r+2 \iint_{\Omega_{R}}\left(\frac{\mu}{\rho}\left|g^{\prime}\right|^{2}-\omega_{1}|g|^{2}\right) \rho r \mathrm{~d} r \mathrm{~d} z .
\end{aligned}
$$

Under the hypothesis (H3), we can see that $a_{1}(u, u)-\omega_{1}^{2}\|u\|^{2}<0$ if $a$ is sufficiently large and the result follows from the Min-Max Principle.

\section{Remark 6.4.}

1) The condition (H3) is satisfied, for example, if $\rho=\rho_{\infty}$ and $\mu_{\infty}-\mu_{0}>0$.

2) In Theorem 6.3 we consider the situation when $\delta_{0}=\mu_{\infty}-\mu_{0}$ is small. In this case there exists always a flexural mode (i.e. harmonic of order $n=1$ ).

\section{Conclusion}

We have studied in this article the spectrum of a self-adjoint operator which models the self-oscillations of an axisymmetric elastic layer considered as a locally perturbed homogeneous layer. In geophysics applications, the earth's layer is more often stratified, then it is important to extend the obtained results to perturbed stratified layer. Finally, we can think of the situation when the perturbation is not axisymmetric (the case where the harmonics in $\theta$ are coupled).

Acknowledgements. This work has been revised while I was visiting the C.M.I. (at the "Université de Provence, France"). I would like to thank Professors M. Ben-Artzi, Y. Dermenjian and P. Joly for their suggestions. 


\section{REFERENCES}

[1] A. Bamberger, Y. Dermenjian and P. Joly, Mathematical analysis of the propagation of elastic guided waves in heterogeneous media. J. Differential Equations 88 (1990) 113-154.

[2] A. Bamberger, P. Joly and M. Kern, Propagation of elastic surface waves along a cylindrical cavity of arbitrary cross section. RAIRO Modél. Math. Anal. Numér. 25 (1991) 1-30.

[3] M. Bouchon and D.P. Schmitt, Full-wave acoustic logging in an irregular borehole. Geophysics 54 (1989) 758-765.

[4] L. Chorfi, Étude mathématique des modes guidés dans un milieu élastique à symétrie de révolution. RAIRO Modél. Math. Anal. Numér. 30 (1996) 299-342.

[5] D.J. Duterte, A.S. Bonnet-Ben Dhia and P. Joly, Mathematical analysis of elastic surface waves in topographic waveguides. $M^{3}$ AS (Math. Models Methods Appl. Sci.) 9 (1999) 755-798.

[6] G. Duvaut, Mécanique des milieux continus. Masson, Paris (1990).

[7] T. Kato, Perturbation Theory for Linear Operators. 2nd edn., Springer-Verlag, New York (1976).

[8] J. Miklowitz, The Theory of Elastic Waves and Wave Guides. North-Holland Publishing Company, Amsterdam, New York, Oxford (1980).

[9] J.A. Nitsche, On Korn's second inequality. RAIRO Anal. Numér. 15 (1981) 237-248.

[10] B. Nkemzi and B. Heinrish, Partial Fourier approximation of the Lamé equation in axisymmetric domains. Math. Methods Appl. Sci. 22 (1999) 1017-1041.

[11] M. Reed and B. Simon, Methods of Modern Mathematical Physics. IV Analysis of Operators. Academic Press, New York, San Francisco, London (1978).

[12] M. Schechter, Operator Methods in Quantum Mechanics. North-Holland Publishing Company, Amsterdam, New York, Oxford (1981).

[13] G. A. Winbow, Seismic sources in open cased boreholes. Geophysics 56 (1991) 1040-1050.

To access this journal online:

www.edpsciences.org 